\title{
Prevention of Fungal Contamination in Plant Tissue Culture Using Cyclic Lipopeptides Secreted by Bacillus amylolique- faciens AB30a
}

\author{
Khushboo Rawal and Hareshkumar Keharia* \\ P. G. Department of Biosciences, Satellite campus, Sardar Patel University, Vadtal Road, Bakrol \\ 388 315, Gujarat, India
}

Key words: Lipopeptides, Bacillus amyloliquefaciens AB30a, Aegle marmelos

\begin{abstract}
Plant tissue culture has revolutionized the field of plant biotechnology. However, there are certain obstacles which overall restrain the output of the plant tissue culturing. One of them is contamination of the tissue culture stock which is a major problem limiting the output. Aegle marmelos (L.) is a medicinal plant whose genotype qualities are maintained through clonal propagation of nodal segment as an explant. It harbors plethora of fungi which curbs the successful in vitro propagation. Chemical fungicide like bavistin is used to prevent the contamination in tissue culture which raises the environmental concerns. Thus, use of microbially derived antifungals can help in preventing fungal growth with benefit of positively impacting the plant growth. Here, authors investigated the use of heat stable lipopeptides which are secondary metabolites derived from Bacillus amyloliquefaciens AB30a for prevention of contamination in tissue culturing of nodal explants of A. marmelos positively impacting its in-vitro propagation.
\end{abstract}

\section{Introduction}

Plant tissue cultures are often being contaminated by microorganisms which come as a resident of explant or as a laboratory contaminant. Various efforts have been made for getting rid of microbial contaminants in plant tissue culture (Harabi et al. 2016, Reed et al.1995). Surface sterilization of the explant to get rid of epiphytic microorganisms is carried out frequently, however there are endophytic organisms which colonize inside the living plant tissue and cause contamination of tissue cultures (Cassells 1991). There are certain tissue cultures which take longer time for the regeneration and if such tissues are bearing an endophytic organism, then it becomes difficult to maintain the contamination free healthy tissue for longer duration (Nair et al. 2014). Incorporation of

*Author for correspondence: <haresh970@gmail.com>. 
antimicrobials is one of the strategies that is employed to maintain contaminant free tissue.

The control of fungal contamination in plant tissue culture is of major concern in comparison to bacterial contamination unlike animal tissue culture. Hence the addition of a fungicide in tissue culture medium becomes a prerequisite in most cases. Certain criteria need to be considered while selecting an antifungal before its incorporation into a medium such as its spectrum of activity, toxicity to the plant tissue, stability and development of resistance (Shields et al. 1984).

Plant fungicides based on synthetic chemicals are extensively used in tissue culture, which cause severe problems due to their toxic and/or carcinogenic nature (Gupta et al. 2007). Consequently, there is an obvious need to search for alternative compounds which are non-toxic and environment friendly. The cyclic lipopeptides produced by several bacterial species are effective against a broad range of fungi at very low concentration (Cawoy et al. 2015). There is no development of resistant microflora against these peptides and they are highly specific (Raaijmakers et al. 2010). Also, it may enhance the plant growth when used in very low concentration (Shafi et al. 2017) and may also induce systemic resistance (Ongena 2007).

Bavistin which is $50 \%(\mathrm{w} / \mathrm{N})$ of carbendazim is routinely used in plant tissue culture for removing epiphytic fungi, however it cannot be incorporated into media for control of endophytic fungi as it is toxic to plant tissue during prolong exposure and at high concentration (Ramseh et al. 2009). Lipopeptides on the other hand overcomes these disadvantages and can be employed in tissue culture medium as a broad-spectrum fungicide for handling the fungal contamination problem (Brotman et al. 2009).

Present study demonstrates the effect of antifungal lipopeptide belonging to family of iturin, fengycin and surfactin which are secreted by Bacillus amyloliquefaciens strain AB30a for in vitro propagation of nodal stem segment of A. marmelos.

\section{Materials and Methods}

The bacterial culture Bacillus amyloliquefaciens AB30a was grown in Luria Bertani broth $(100 \mathrm{ml}$ in $250 \mathrm{ml})$ flask for $120 \mathrm{hrs}$ under shaking condition of $150 \mathrm{rpm}$ at $30^{\circ} \mathrm{C}$. The culture broth at the end of fermentation period was centrifuged at 10,000 rpm for $10 \mathrm{~min}$ to get rid of cells. The supernatant was then acidified using $2 \mathrm{~N} \mathrm{HCl}$ up to a $\mathrm{pH}$ of 2 . The precipitation was allowed to occur under cooling condition overnight. Precipitates were recovered by centrifugation. The acid precipitates were solubilized in methanol and the methanol extract was dried by evaporation and the dried extract was used as crude lipopeptide preparation. The in vitro antifungal activity of the crude methanol extract was tested against different Phyto-pathogenic fungi by agar well diffusion assay. 
Thermostability of the crude lipopeptide extract was tested by autoclaving the crude methanol extract at $121^{\circ} \mathrm{C}$ for $15 \mathrm{~min}$. The autoclaved extract was resuspended in respective amount of methanol and tested for antifungal activity using bioautograph.

The autoclaved and unautoclaved lipopeptide extracts were separated by TLC on silica plate using chloroform : methanol : water :: $65: 30: 5$ as solvent system and bioautography was performed against the test fungi Aspergillus niger by taking $100 \mathrm{ul}$ fungal spore suspension and inoculating it in $0.8 \%$ sterile molten potato dextrose soft agar and overlaying it on silica plate where the extract was resolved using TLC the plate was incubated under moist condition and observed for fungal growth inhibition.

The young twig was collected from the mature plant of A. marmelos (L.) from the garden of P. G. Department of Biosciences, Sardar Patel University, Anand, Gujarat, India. The nodal stem of $A$. marmelos was excised and used as explants.

The excised nodal explants were surface cleaned by washing under running tap water for $5 \mathrm{~min}$. The washed explants were then dipped in 1\% HI Clean (Liquid soap, Himedia) solution for $10 \mathrm{~min}$ by constant stirring. Further cleaning of the explant was done by adding 2 - 3 drops of Tween-20 solution and washed for $20 \mathrm{~min}$. The cleaned explants were then brought into laminar air flow and transferred into sterile distilled water. The explants were then dipped in to sterile $0.1 \%(\mathrm{w} / \mathrm{N}) \mathrm{HgCl}_{2}$ solution and treated for $2 \mathrm{~min}$ and then subjected to sterile distill water wash for 3 - 4 times to remove traces of mercuric chloride. The surface sterilized nodal explants were then inoculated into MS medium with plant hormones.

For carbendazim treatment the surface cleaned explants were dipped in carbendazim solution for $20 \mathrm{~min}$ and then repeatedly washed with sterile distilled water. The explants were then treated with $0.1 \%(\mathrm{w} / \mathrm{N}) \mathrm{HgCl}_{2}$ solution for $2 \mathrm{~min}$ and then washed 5 - 6 times with sterile distilled water. The used water from the last wash was streaked on to Potato dextrose agar medium to ensure the surface sterility of the explant. When lipopeptide extract was used as a fungicide, the explants were surface sterilized with $\mathrm{HgCl}_{2}$ first and then washed and transferred to lipopeptides solution and treated for $20 \mathrm{~min}$. The lipopeptides treated explants were immediately transferred to tissue culture medium without further washing. Carbendazim was used as a control fungicide and crude lipopeptide was used as a test fungicide and both the fungicides were used at 50 and 100 $\mu \mathrm{g} / \mathrm{ml}$ concentration.

To study the effect of fungicidal agent experiment included three treatment groups: Only explant is treated with lipopeptide or carbendazim (used as control), explant treated with fungicide as well as augmented in plant tissue culture media, surface sterilized explant directly incorporated into tissue culture medium containing fungicide treated explants were transferred into respectively labelled tubes aseptically with emerging node facing in upright position. There were 10 replicates of each treatment. 
The results were tabulated as contamination inhibition (\%) calculated by number of explants contaminated total number of explants per treatment.

The fungal contaminants which appeared after prolong incubation were observed after staining with lactophenol cotton blue and visualized using light microscopy technique at 400x magnification.

\section{Results and Discussion}

The antifungal lipopeptides extracted from Bacillus amyloliquefaciens AB30a belonged to the family of iturin, fengycin and surfactin. Crude lipopeptide (LP) mixture was examined in vitro for fungal growth inhibition which was found to inhibit a number of plant pathogenic fungi (Fig. 1) viz, Aspergillus niger, Fusarium sp., Alternaria sp. Thus, these lipopeptides which are broad spectrum antifungal can also be incorporated in tissue culture media for testing its efficacy in inhibiting the fungal contamination in tissue culture.

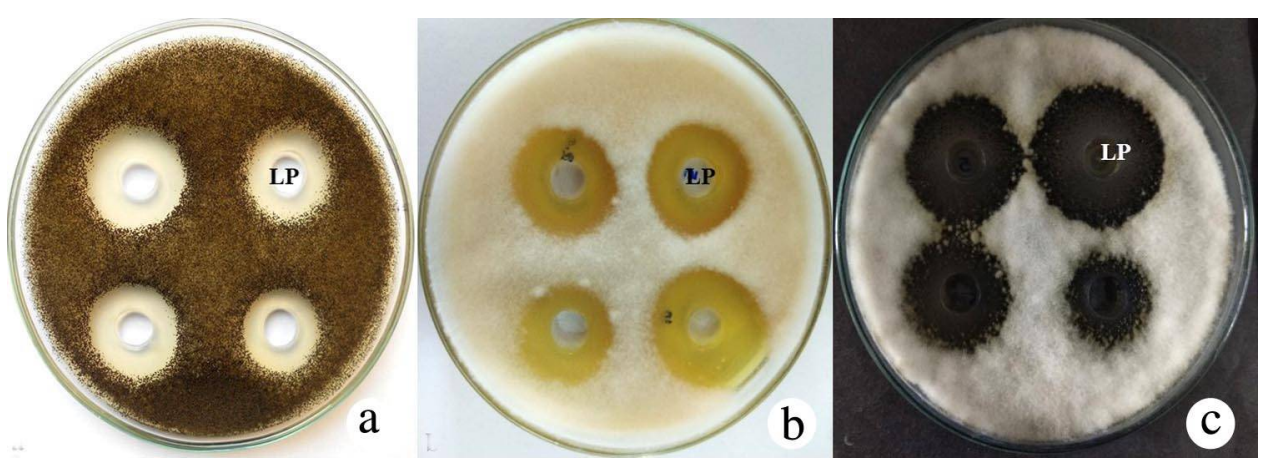

Fig. 1. Antagonistic activity of crude lipopeptide from B. amyloliquefaciens AB30a in agar well diffusion assay against a. Aspergillus niger, b. Fusarium sp., c. Alternaria sp.

The fungicidal lipopeptides retained antifungal activity even after autoclaving at $121^{\circ} \mathrm{C}$ for $15 \mathrm{~min}$ which is the general method followed for media sterilization. The thermal stability of lipopeptides is depicted in Fig. 2. Owing to their thermostable nature and ability to withstand autoclaving, these lipopeptides can become a promising agent for the tissue culture application as they can be incorporated into media prior to sterilization, making it convenient and cost-effective. Synthetic antifungal agents are not always thermostable and are to be sterilized by filter sterilization for augmentation in to autoclaved plant tissue culture media, thus making it cumbersome and further increasing the chances of contamination.

A. marmelos possesses a number of endophytic fungi. Different fungal species have been reported to colonize bark, root and leaf tissues (Gond et al. 2007). Aspergillus, Rhizoctonia, Stenella, Fusarium and Alternaria are the major genera which inhabit different 
plant parts (Gawas et al. 2010) because of which its in vitro clonal propagation becomes difficult and that is why it becomes mandatory to incorporate fungicidal treatment during its tissue culturing. The treatment of explants with antifungal agent can help in eliminating the epiphytic fungi but not the growth of endophytic fungi and that is why the incorporation of antifungal agents in plant tissue culture medium are employed for inhibiting the endophytic fungal growth. The synthetic fungicides which are routinely used can only be employed for surface sterilization of the explants, their incorporation into media for controlling endophytic fungal growth may lead to toxicity to explant. The cyclic lipopeptides on the other hand are non-toxic and are heat stable.

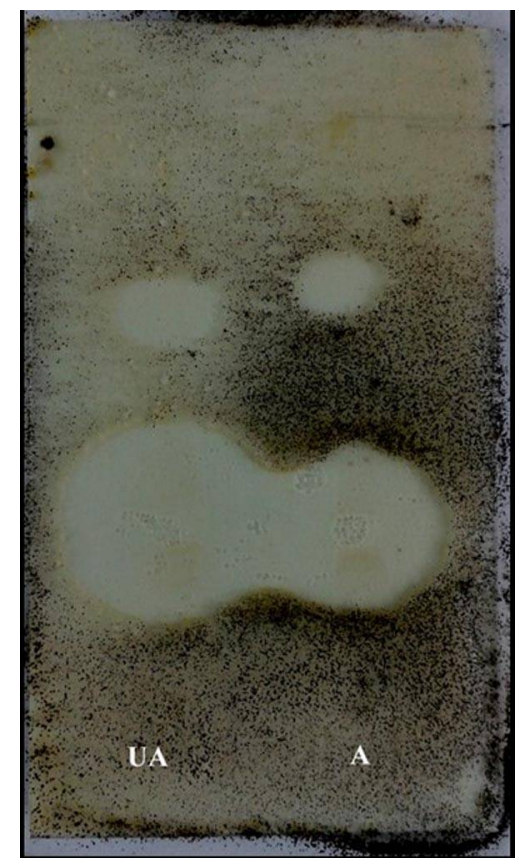

Fig. 2. The silica gel thin layer bioautography showing the inhibition of Aspergillus niger by unautoclaved (UA) and autoclaved (A) antifungal extract.

Thus, to elucidate the effect of lipopeptide as antifungal agent in tissue culture, the antifungal agent was incorporated in the tissue culture medium and also the explant was treated with the lipopeptide and inoculated in sterile tissue culture medium, combination of both the treatments was also used. The results depicted in Fig. 3 indicate the percentage inhibition of contamination observed after different treatments.

The lipopeptide at $50 \mu \mathrm{g} / \mathrm{ml}$ concentration did not show reduction in inhibition of fungal contaminants irrespective of the treatment methodology, whereas the lipopeptide at $100 \mu \mathrm{g} / \mathrm{ml}$ concentration was found to significantly inhibit the fungal growth. The treatment of explants with the lipopeptide alone does not inhibit the fungal contaminant 
effectively, however incorporation of lipopeptide extract in to the media is found to be useful, which suggests that the prolong exposure can completely inhibit the proliferation of endophytic fungi residing in A. marmelos (Fig. 4). In comparison to carbendazim fungicide, lipopeptide seems to be more effective antifungal agent for prevention of fungal growth in plant tissue culture.

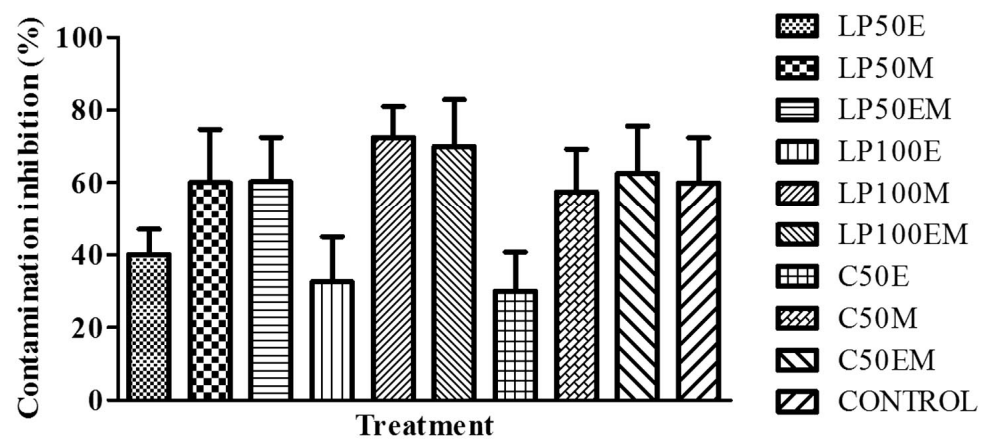

Fig. 3. Percentage inhibition of fungal contamination after different treatments. Results are average of independent observations and error bars indicate the standard error from mean.
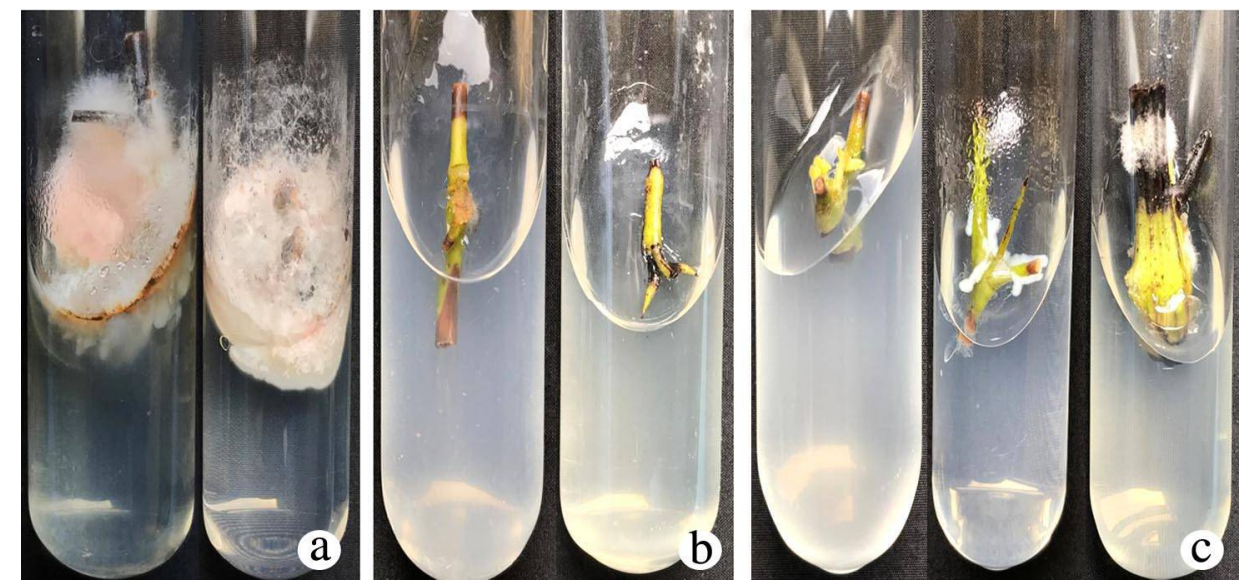

Fig. 4. Influence of lipopeptide treatment to explants on contamination in plant tissue culture: (a) Control without any treatment, (b) carbendazim treated explants and (c) lipopeptide treated explants.

The other advantage of using lipopeptide is its non-toxicity to the explant and also it does not hinder the regeneration and differentiation process of plant tissue. At lower concentration of lipopeptide it is known to enhance the plant growth (Fig. 4). Nair et al. (2016) studied the effect of fengycin lipopeptide on the seed germination of Vigna radiata and Oryzae sativa in vitro wherein they observed that the incorporation of the fengycin derived from the marine endophytic Bacillus sp. delayed the growth of seeds without it 
getting decayed and concluded that the stereochemistry of amino acid residues in the lipopeptide is extremely important for the activity. Cyclic lipopeptides are microheterogenous structure (Ueda et al. 1984) and use of the crude lipopeptide preparation would be more effective against more than one group of fungal contaminants, thereby enhancing its spectrum. Chemical fungicide on the other hand might have toxic effect on prolong exposure to the tissue. Nagi et al. (2005) studied the effect of incorporation of different fungicide amongst which miconazole, potassium sorbate, cycloheximide and copper sulphate inhibited the growth of the contaminating yeast Rhodotorula slooffiae but were phytotoxic to the apple shoot.

The contaminated explants were microscopically observed for identifying the type of fungal contamination (Fig. 4) and it was observed that majority of the contaminated explants were infected with Fusarium and Alternaria sp. of fungi (Fig. 5). The occurrence of Alternaria, Stenella, Aspergillus, Rhizoctonia, Fusarium, Xylaria as endophytes in A. marmelos have been reported by Gond et al. (2007).
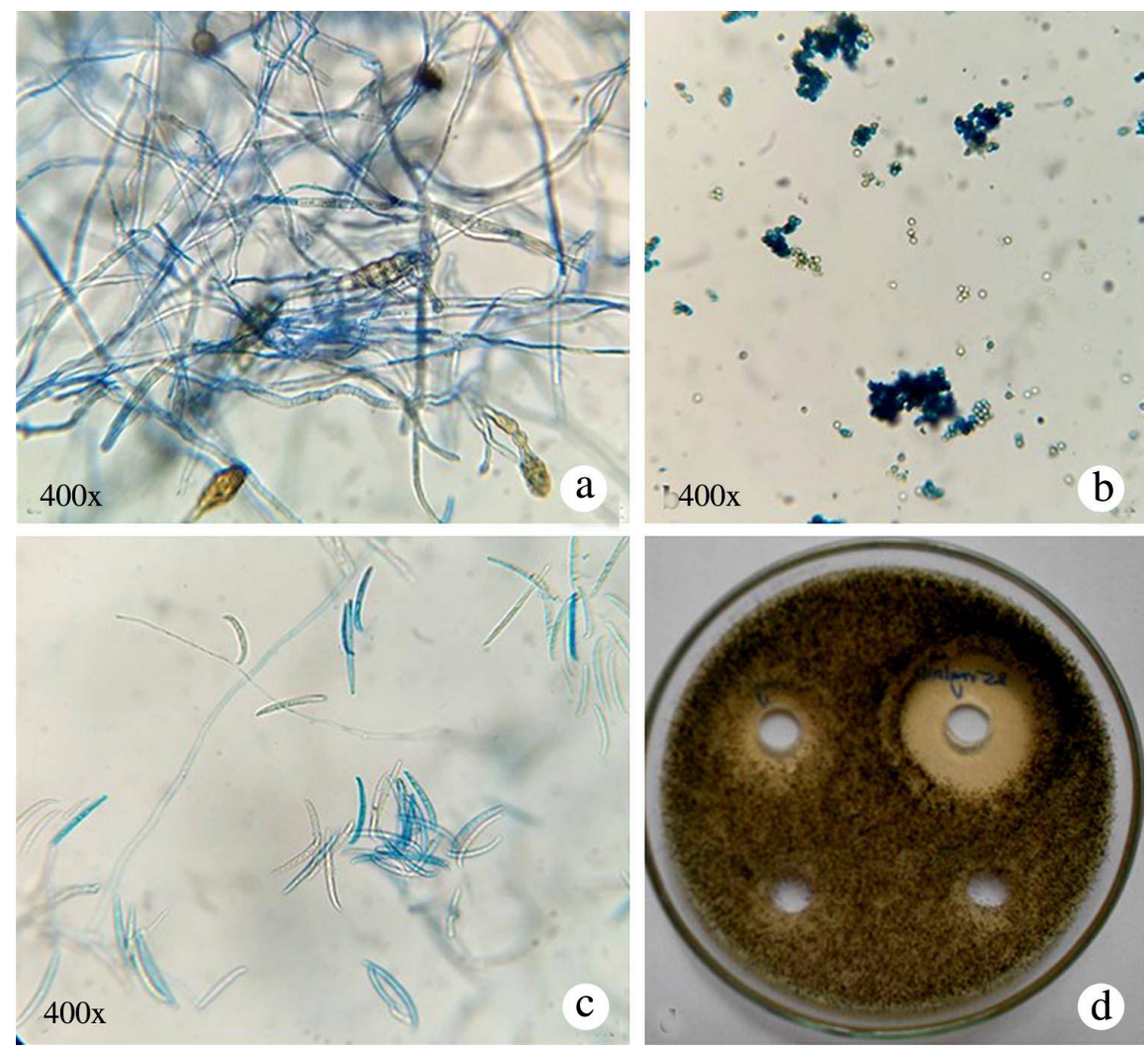

Fig. 5. Microscopic observation of fungal contamination in explants showing spores of (a) Alternaria, (b) Aspergillus and (c) Fusarium, (d) agar diffusion bioassay of crude lipopeptide extract against Aspergillus niger. 
In vitro inhibition assays on Fusarium and Alternaria were carried out and it did not get inhibited at the concentration used for performing the tissue culture experiments, but increasing the lipopeptide concentration did inhibit the fungal growth so by taking higher concentration of lipopeptide can help in inhibiting the endophytic fungi which are the major causes of contamination.

\section{Acknowledgements}

First author (KR) gratefully acknowledges Department of Science and Technology (DST), New Delhi, for the financial support extended in the form of INSPIRE Fellowship (IF130977) and authors also gratefully acknowledge the funds received by University Grants Commission, Government of India, New Delhi under Centre of Advanced Study to the Department. They acknowledge the help of Mr. Dinesh Vasava, Assistant Professor at Department of Biosciences, Sardar Patel University for helping in setting up of Tissue Culture experiments.

\section{References}

Brotman Y, Makovitzki A, Shai Y, Chet I and Viterbo A (2009) Synthetic ultrashort cationic lipopeptides induce systemic plant defense responses against bacterial and fungal pathogens. App and Environmental Microbiology 75(16): 5373-5379.

Cassells AC (1991) Problems in tissue culture: culture contamination. In: Debergh P.C., Zimmerman R.H. (eds) Micropropagation., Springer, Dordrecht, pp. 31-44.

Cawoy H, Debois D, Franzil L, De Pauw E, Thonart P and Ongena M (2015) Lipopeptides as main ingredients for inhibition of fungal phytopathogens by Bacillus subtilis/amyloliquefaciens. Microbial Biotechnology 8(2): 281-295.

Gawas SP and Bhat DJ (2010) Mycoflora associated with Aegle marmelos, a medicinal plant of the forests of Western Ghats, India. Kavaka 37-46.

Gond SK, Verma VC, Kumar A, Kumar V and Kharwar RN (2007) Study of endophytic fungal community from different parts of Aegle marmelos Correae (Rutaceae) from Varanasi (India). World Journal of Microbiology and Biotechnology 23(10): 1371-1375.

Gupta PK (2018) Toxicity of fungicides. In: Veterinary Toxicology (3rd). pp. 569-580.

Harabi A, Bouzerara F, Foughali L, Boudaira B, Guechi A and Brihi N (2016) Elaboration and characterization of low-cost ceramics microfiltration membranes applied to the sterilization of plant tissue culture media. Journal of the Taiwan Institute of Chemical Engineers 59: 79-85.

Nagy JK, Sule S and Sampaio JP (2005) Apple tissue culture contamination by Rhodotorula spp.: identification and prevention. In Vitro Cellular \& Developmental Biology-Plant 41(4): 520-524.

Nair D, Vanuopadath M, Nair BG, Pai JG and Nair SS (2016) Identification and characterization of a library of surfactins and fengycins from a marine endophytic Bacillus sp. Journal of Basic Microbiology 56(11): 1159-1172.

Nair DN and Padmavathy S (2014) Impact of endophytic microorganisms on plants, environment and humans. The Scientific World Journal. 2014:1-11. 
Ongena M, JourdanE, Adam A, Paquot M, Brans A, Joris B and Thonart P (2007) Surfactin and fengycin lipopeptides of Bacillus subtilis as elicitors of induced systemic resistance in plants. Environmental Microbiology 9(4): 1084-1090.

Raaijmakers JM, De Bruijn I, Nybroe O and Ongena M (2010) Natural functions of lipopeptides from Bacillus and Pseudomonas: more than surfactants and antibiotics. FEMS microbiology Reviews 34(6): 1037-1062.

Ramseh M, Marx R, Mathan G and Pandian SK (2009) Effect of bavistin on in vitro plant conversion from encapsulated unimodal microcuttings of micro-propagated Bacopa monnieri (L.) An ayurvedic herb. Journal of Environmental Biology 30(3): 441-444.

Reed BM and Tanprasert P (1995) Detection and control of bacterial contaminants of plant tissue cultures. A review of recent literature. Plant Tissue Culture and Biotechnology 1(3):137-142.

Shafi J, Tian H and Ji M (2017) Bacillus species as versatile weapons for plant pathogens: A review. Biotechnology \& Biotechnological Equipment 31(3): 446-459.

Shields R, Robinson SJ and Anslow PA (1984) Use of fungicides in plant tissue culture. Plant Cell Reports 3(1): 33-36.

Ueda T, Sada I and Kato T and Izumiya N (1985) Cyclic peptides. XIX. Synthesis, conformation, and biological activity of cyclo (-Pro-Val-Pro-Val) with 1-1-1-1 and 1-d-1-d sequences. International Journal of Peptide and Protein Research 25(5): 475-480. 